\title{
Costo-efectividad de la colecistectomía laparoscópica y de la abierta en una muestra de población colombiana
}

Roosevelt Fajardo', José Ignacio Valenzuela ${ }^{1,2}$, Sandra Catalina Olaya ${ }^{3}$, Gustavo Quintero², Gabriel Carrasquilla ${ }^{3}$, Carlos Eduardo Pinzón", Catalina López¹, Juan Camilo Ramírez ${ }^{5}$

\author{
1 Centro de Innovación y Educación en Salud, Fundación Santa Fe de Bogotá, Bogotá, D.C., Colombia \\ 2 Escuela de Medicina, Universidad del Rosario, Bogotá, D.C., Colombia \\ ${ }^{3}$ Centro de Estudios e Investigación en Salud, Fundación Santa Fe de Bogotá, Bogotá, D.C., Colombia \\ ${ }^{4}$ Colaboración Cochrane, Bogotá, D.C., Colombia \\ 5 Hospital de San José, Fundación Universitaria de Ciencias de la Salud, Bogotá, D.C., Colombia
}

Introducción. La colecistectomía ha sido objeto de varios estudios clínicos y de costos a nivel mundial.

Objetivo. Evaluar el costo-efectividad de la colecistectomía abierta y de la laparoscópica desde la perspectiva del tercer pagador.

Materiales y métodos. Se llevó a cabo un estudio de costo-efectividad en dos hospitales de Bogotá. Se obtuvieron los datos de efectividad de las cirugías a partir de un estudio de cohortes, y se obtuvo en forma retrospectiva el tipo de colecistectomía y en forma prospectiva, el resultado.

Se incluyeron 376 pacientes intervenidos por colecistitis o litiasis de mayo de 2005 a junio de 2006; 156 pacientes fueron intervenidos por colecistectomía abierta y 220 por laparoscopia.

Los resultados que se analizaron fueron los siguientes: frecuencia de complicaciones, estancia hospitalaria, reincorporación a las labores cotidianas y duración de la cirugía. Los costos se recolectaron según el tipo de estudio económico y sólo se incluyeron los costos directos.

Posteriormente, se planteó un estudio de costo-efectividad a un año desde la perspectiva del tercer pagador; se propuso un modelo de árbol de decisiones y se calcularon las razones de incremento de costo-efectividad para cada uno de los desenlaces propuestos.

Resultados. Los resultados de la colecistectomía abierta frente a la laparoscópica fueron: OR ajustado de complicaciones: 2,02 ( $\mathrm{IC}_{95 \%}$ 0,94-4,37); conversión quirúrgica (tasa): 3,2\%; estancia: 2,2 Vs. 1,6, $p=0,003$; reincorporación a cotidianidad: 32,5 Vs. 9,6, $p<0,001$; duración quirúrgica: 22 minutos $(p<0,001)$ menor en la colecistectomía abierta; desde la perspectiva del tercer pagador, costo promedio: US\$ 995 Vs. US\$ 1.048; incremento del costo-efectividad: US \$ 7,4 favoreciendo la laparoscópica; desde la perspectiva paciente, costo promedio: US\$ 53.2 Vs. US\$ 104,8; incremento del costo-efectividad: US\$ -7.3 favoreciendo la laparoscópica; cero mortalidad. La variable que más impactó tiene en la toma de decisiones en términos de costos es el costo del procedimiento quirúrgico.

Conclusiones. Hubo mayor estancia hospitalaria en la colecistectomía abierta frente a la mayor duración quirúrgica en la laparoscópica; en el costo directo de la laparoscópica: menor para IPS y pacientes; y en costo-efectividad equiparable para ambos procedimientos.

Palabras clave: colecistectomía laparoscópica, análisis costo-beneficio, economía de la salud, Colombia.

\section{Cost-effectiveness of laparoscopic versus open cholecystectomy}

Introduction. Cholecystectomy has been the subject of several clinical and cost comparison studies. Objective. The results of open or laparoscopy cholecystectomy were compared in terms of cost and effectiveness from the perspective of healthcare institutions and from that of the patients.

Materials and methods. The cost-effectiveness study was undertaken at two university hospitals in Bogotá, Colombia. The approach was to select the type of cholecystectomy retrospectively and then assess the result prospectively. The cost analysis used the combined approach of micro-costs and daily average cost. Patient resource consumption was gathered from the time of surgery room entry to time of discharge. A sample of 376 patients with cholelithiasis/cystitis (May 2005-June 2006) was selected-156 underwent open cholecystectomy and 220 underwent laparoscopic cholecystectomy. The following data were tabulated: (1) frequency of complications and mortality, post-surgical hospital stay, (2) reincorporation to daily activities, (3) surgery duration, (4) direct medical costs, (5) costs to the patient, and (6) mean and incremental cost-effectiveness ratios.

Results. Frequency of complications was $13.5 \%$ for open cholecystectomy and $6.4 \%$ for laparoscopic cholecystectomy $(p=0.02)$; hospital stay was longer in open cholecystectomy than in laparoscopic 
cholecystectomy ( $p=0.003$ ) as well as the reincorporation to daily activities reported by the patients $(p<0.001)$. The duration of open cholecystectomy was $22 \mathrm{~min}$ longer than laparoscopic cholecystectomy $(p<0.001)$. The average cost of laparoscopic cholecystectomy was lower than open cholecystectomy and laparoscopic cholecystectomywas more cost-effective than open cholecystectomy (US\$ 995 vs. US $\$ 1,048$, respectively). The patient out-of-pocket expenses were greater in open cholecystectomy compared to laparoscopic cholecystectomy $(p=0.015)$. Mortality was zero.

Conclusions: The open laparoscopy procedure was associated with longer hospital stays, whereas the cholecystectomy procedure required a longer surgical duration. The direct cost of the latter was lower for both for the healthcare institution and patients. The cost-effectiveness for both procedures was comparable.

Key words: Cholecystectomy, laparoscopic; cost-benefit analysis, health economics, Colombia.

La colecistectomía laparoscópica, técnica quirúrgica introducida a mediados de la década de los ochenta para el tratamiento de la enfermedad litiásica vesicular (1), ha sido objeto de varios estudios en que la evalúan en términos de resultados clínicos y de costos, comparándola con el método tradicional, la colecistectomía abierta. Muchos de estos análisis favorecen ampliamente la técnica laparoscópica (2-6) y, en efecto, su uso se ha generalizado en el ámbito mundial; se ha convertido en el procedimiento quirúrgico de elección para el tratamiento de dicha enfermedad y ha demostrado ventajas clínicas, tales como la reducción en la morbilidad y mortalidad, y económicas, al representar menores costos para los pagadores del sistema de salud en general.

A pesar de la experiencia internacional, hasta diciembre de 2005 en Colombia este procedimiento estuvo excluido de los planes de beneficios ofrecidos dentro del Sistema General de Seguridad Social en Salud, y hoy, parte de la población aún no cuenta con acceso al mismo, argumentándose mayores costos frente a la colecistectomía abierta. Además, las condiciones de negociación entre aseguradores y prestadores son las que definen los procedimientos y técnicas que pueden practicarse, teniendo en cuenta fundamentalmente la tarifa (7), la cual establece un precio mayor para la técnica laparoscópica.

Hasta el momento, se tiene conocimiento de dos estudios llevados a cabo en el país, en los cuales se analiza el costo-efectividad de la técnica laparoscópica. Uno es el de Moore, etal. (7), quienes por medio de un seguimiento a pacientes sometidos

\footnotetext{
Correspondencia:

José Ignacio Valenzuela, Centro de Innovación y Educación en Salud, Fundación Santa Fe de Bogotá, Carrera 7B № 123-90, piso 4, Bogotá, D.C., Colombia.

Teléfono: (571) 603 0303; extensión 5721; fax: (571) 2146668 jose.valenzuela@fsfb.edu.co

Recibido: 15/02/10; aceptado:07/07/11
}

a esta técnica en un centro de cirugía ambulatoria, midieron su efectividad y la compararon con la información encontrada en la literatura científica (revisiones sistemáticas y metaanálisis) sobre laparoscopia con hospitalización y sobre la técnica abierta. Con base en esta revisión, se partió de niveles similares de efectividad en los tres casos y se adoptó un modelo de minimización de costos, según el cual se concluyó que es factible realizar laparoscopia ambulatoria en Colombia a precios accesibles y al alcance de la comunidad (7). El segundo es el de Olaya y Carrasquilla (8), un modelo de algoritmo de decisión del costo-efectividad de la técnica laparoscópica comparada con la abierta, en el que se evaluó la probabilidad de complicación y mortalidad, calculada por medio de un metaanálisis, y el costo de ambos procedimientos con base en las tarifas establecidas para el país en 2004. En el estudio se favorece la técnica laparoscópica en cuánto a costo y a efectividad.

Con el objetivo de tener información local sobre efectividad y costo de ambas técnicas, en el presente estudio, por medio de una evaluación económica desde el punto de vista del paciente y de la IPS, se compararon los costos directos y la efectividad -en términos de complicaciones evitadas- de la colecistectomía laparoscópica frente a la abierta, en una muestra de la población colombiana.

\section{Materiales y métodos}

Se hizo un estudio de costo-efectividad con la perspectiva del tercer pagador, durante el término de un año, para evaluar los resultados de interés y se incluyeron sólo los costos directos asociados a la intervención. Para obtener los datos de efectividad para el modelo, se llevó a cabo un estudio observacional de cohortes, en el que la exposición (tipo de procedimiento) se obtuvo retrospectivamente (después de que el médico tratante había tomado la decisión sobre el tipo de intervención) y el resultado se tomó de manera 
prospectiva. Se seleccionaron los pacientes con enfermedad vesicular sometidos a colecistectomía laparoscópica o abierta, en dos hospitales universitarios de Bogotá (Hospital Universitario de la Fundación Santa Fe de Bogotá y Hospital de San José), desde el momento de inicio del estudio y hasta completar el tamaño de muestra requerido.

De acuerdo con la literatura científica sobre el tema (9) y la opinión de expertos, se tomó como referencia una frecuencia de complicaciones de colecistectomía abierta de $3 \%$ y de la laparoscópica de $1 \%$. Con un nivel de significancia de $5 \%$, poder de $80 \%$ y teniendo en cuenta un nivel de $25 \%$ de falta de respuesta o rechazo, la muestra calculada fue de 210 pacientes en cada grupo. Se hizo un análisis interno con el objetivo de evaluar la tasa de complicaciones en el grupo de cirugía laparoscópica y en el de cirugía abierta, y se encontró una tasa de complicaciones de 13,4\% y de 6,36\%, respectivamente; se calculó nuevamente el tamaño de la muestra bajo estos parámetros y los supuestos anteriormente mencionados, lo cual arrojó un resultado de 350 sujetos, es decir, 175 sujetos por grupo.

Los criterios de inclusión fueron pacientes hospitalizados en los dos centros participantes, para colecistectomía electiva o de urgencias, de cualquier edad, sexo y gravedad en su estado de salud al ingresar al hospital, y que aceptaran participar en el estudio.

Después de que los pacientes fueron intervenidos, se les indagó sobre su interés en participar en el estudio y, posterior a su aceptación y firma de un consentimiento informado, se procedió a recolectar la información. Se excluyeron los pacientes sometidos a otro tipo de procedimiento quirúrgico durante la misma hospitalización en la que se practicó la colecistectomía y los intervenidos por los investigadores del estudio.

La información general del paciente que se obtuvo fue: edad, sexo, tipo de afiliación, carácter de la cirugía (urgencia o programada), ASA (clasificación que estima el riesgo de la anestesia) (10), ocupación e información referente al diagnóstico.

Las principales medidas de resultados fueron: frecuencia de complicaciones y mortalidad, duración de la estancia hospitalaria, duración de la cirugía, incapacidad y costos directos. Se adoptó la definición de costo directo de Gold, et al., según la cual incluye todos los bienes, servicios y otros recursos consumidos en la provisión de una intervención, incluyendo los efectos secundarios $u$ otras consecuencias presentes o futuras ligadas a ella. Se dividieron en costos directos de la atención médica y en costos directos no relacionados con ella (como los costos del tiempo gastado por los pacientes, familiares o de productividad, asociados al tratamiento), para la IPS y para los pacientes. La incapacidad se midió en términos subjetivos, reportada por el paciente, y normativos, certificada por el médico tratante. Las fuentes de información fueron la historia clínica, los registros de costos y la facturación detallada de los hospitales y los pacientes.

Para el componente económico se estimaron sólo los costos médicos directos asociados a la intervención, por metodología de "microcosteo", desde la perspectiva del tercer pagador $(11,12)$, de acuerdo con el cual se recolectó información de los recursos utilizados por cada paciente desde el momento de su entrada al quirófano para ser intervenido, hasta el momento de su salida del hospital.

El nivel de detalle varió según la información disponible. Los medicamentos, suministros, imágenes diagnósticas, exámenes de laboratorio, estudio anatomopatológico, procedimientos médicos y personal médico que participó en la cirugía (cirujano, anestesiólogo y ayudantes de cirugía), se trabajaron con costo unitario, mientras que se usó un costo promedio de día de estancia en piso, en la unidad de cuidados intensivos y derechos de sala. En estos últimos quedaron incluidos los servicios de enfermería e instrumentación quirúrgica.

En cuanto a equipos, se calculó individualmente el costo para el de laparoscopia y para el de la técnica abierta (incluyendo el instrumental quirúrgico), asumiéndose que todos los pacientes sometidos a un mismo procedimiento tienen la misma necesidad de equipos durante la cirugía. Para determinar el porcentaje de uso de trocares desechables en laparoscopia, se diligenció un formato durante la intervención quirúrgica que indagaba sobre el uso de elementos desechables.

En cuanto a los costos de medicamentos y suministros, se utilizó el costo de adquisición en el mercado de cada hospital. El costo del día de estancia en el pabellón y en la unidad de cuidados intensivos, se calculó de acuerdo con el costo total anual y la estadística de ocupación día-cama durante el año: tasa de ocupación anual de $92 \%$ y $85 \%$, para pabellón y unidad de cuidados intensivos respectivamente. Por ausencia de datos históricos de ocupación en los hospitales, se trabajó con el promedio de ocupación de los dos últimos años. 
El costo por hora en sala de cirugía se calculó según el costo generado en la unidad quirúrgica durante el año y el promedio de ocupación de las salas disponibles (tasa de ocupación del $80 \%$ ).

En cuanto a equipos, se estimó el costo de depreciación anual en línea recta de acuerdo con la vida útil de cada equipo y éste se dividió por el número de cirugías durante el año, para obtener un costo de equipos por paciente. No se aplicó tasa de descuento, puesto que las intervenciones son evaluadas en un corto período. El costo de cirujanos, ayudantes y anestesiólogos, corresponde al de honorarios pagados por la cirugía.

Para homologar los costos de adquisición en los dos hospitales y utilizar el mismo valor de los recursos en todos los pacientes, se hizo un ajuste promediando el costo de los ítems utilizados por ambos hospitales y se usó este valor promedio para todos los pacientes, independientemente del hospital en que hubieran sido intervenidos. El costo por paciente se calculó multiplicando el valor de cada uno de los ítems mencionados por la cantidad utilizada en cada paciente. Se trabajó con precios de mercado (SOAT, ISS) a diciembre de 2005 y los costos finales se presentan en pesos constantes de diciembre 2006, ajustados por el índice de precios al consumidor (IPC). No se aplicaron tasas de descuento, dado el horizonte de tiempo propuesto.

En cuanto a la estimación de costos directos para el paciente, se indagó por el gasto de bolsillo y el tiempo de reincorporación a las tareas cotidianas después de la intervención. Para ello, hubo comunicación telefónica con los pacientes a los 15 y 30 días después de la cirugía, indagando sobre el gasto efectuado en medicamentos, transporte, consultas médicas, elementos de curación, asistencia médica o de enfermería en el hogar, apoyo en tareas domésticas por incapacidad, cuidado por parte de familiares o amigos, gastos adicionales y tiempo de reincorporación a tareas cotidianas.

Se calculó el costo de oportunidad del tiempo utilizado en el cuidado de los pacientes por parte de los familiares durante la recuperación. Para ello, se hizo una aproximación multiplicando el salario mínimo mensual legal diario por el número de días perdidos de trabajo o de actividades de ocio. No se calculó el costo del tiempo gastado por los pacientes al recibir el tratamiento: tiempo de viaje, tiempo de espera en citas de control y tiempo gastado en recibir el tratamiento aparte de la incapacidad (12), considerándose que las alternativas analizadas comprenden gastos de tiempo similares y que, por ende, su inclusión tendría un efecto pequeño sobre los resultados. Los costos estructurales y administrativos se contemplaron dentro de cada una de las actividades descritas en la intervención, diagnóstico y seguimiento, en cada uno de los casos.

\section{Análisis estadístico}

Las variables continuas se describieron usando media y desviación estándar, y las categóricas, utilizando frecuencias. Se hizo un análisis bivariado utilizando las pruebas de ji al cuadrado y $\mathrm{t}$ de Student,con un nivel de significancia de $5 \%$. Posteriormente, se calculó la razón de momios (odds ratio, OR) crudo y ajustado de complicaciones y la diferencia en medias de días de estancia hospitalaria, incapacidad y tiempo quirúrgico, con sus respectivos intervalos de confianza. En cuanto a la variable "complicaciones", se ajustó un modelo de regresión logística para observar la relación entre la variable "procedimiento" (exposición) y las complicaciones (variable dependiente), ajustando por las variables de control, a saber, edad, sexo, ASA y carácter de la cirugía (urgencia o programada). Además, se hizo un proceso por etapas (stepwise) para determinar la asociación entre las variables independientes y la variable complicaciones. En cuanto a estancia y duración de la cirugía, se utilizó un modelo sólido de regresión, ajustando por las mismas variables de control. Se calcularon medidas de estancia y duración de la cirugía, ajustadas por las variables de confusión que tuvieron asociación significativa con su variabilidad. Para todos los análisis, se utilizó un valor alfa de $5 \%$. El análisis se efectuó en Stata $9{ }^{\circledR}$.

\section{Análisis de costo-efectividad}

Se hizo el análisis desde la perspectiva del tercer pagador y en algunos resultados se incluyeron los costos asociados al compromiso del bolsillo del paciente. Se consideró como la principal medida de efectividad, el estado del paciente después de la cirugía: sano o sin complicaciones.

El costo promedio por procedimiento se estimó por medio de un algoritmo de decisiones. Para ello, se partió de una condición inicial (colecistitis, colelitiasis), de la que se desprendían dos ramas de decisión: colecistectomía abierta y laparoscópica. A partir de la intervención, los pacientes podían experimentar cualquiera de dos estados en el caso del procedimiento abierto: 1) se complicó la cirugía 


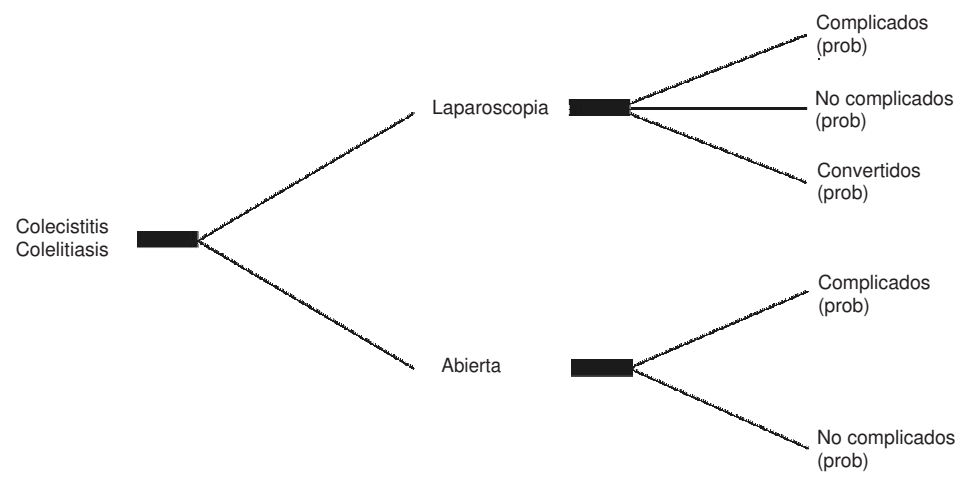

Figura 1. Algoritmo de decisión

o se presentaron complicaciones posoperatorias, lo que en algunos casos generó más días de estancia hospitalaria o alguna reintervención quirúrgica, y 2) no se presentaron complicaciones posoperatorias (figura 1). En el caso de la laparoscopia, además de las anteriores, existía una opción adicional correspondiente a cirugías convertidas de laparoscopia a procedimiento abierto. A cada uno de estos estados se le asignó una frecuencia y un costo, lo que permitió establecer un costo promedio por cirugía.

Además, se calculó la medida de incremento de costo-efectividad (13) como el cociente entre la diferencia en costos y en efectividad entre las dos técnicas evaluadas:

$$
C E I=\frac{C_{i}-C_{j}}{E_{i}-E_{j}}
$$

Se consideró una medida costo-efectiva, toda aquella que estuviera por debajo del producto interno bruto (PIB) per cápita del país.

\section{Análisis de sensibilidad}

Con el objetivo de valorar la solidez de la estimación realizada (11), se hizo un análisis de sensibilidad univariante o de una vía (14), contemplando diferentes circunstancias, según el cual se modificó solamente el valor de una de las variables, en este caso, los costos, utilizando el rango obtenido en los pacientes del estudio.

Para ello, se plantearon cuatro posibilidades iniciales, de la siguiente manera:

1) la mejor situación para el procedimiento abierto, comparado con el peor en el procedimiento laparoscópico,

2) la mejor situación para el procedimiento laparoscópico, comparado con la peor en el procedimiento abierto,
3) la peor situación para ambos procedimientos, y

4) la mejor situación para ambos procedimientos (15).

Se planteó una quinta posibilidad utilizando la mediana del costo y, en vista de que el uso de trocares desechables es señalado como un factor que incrementaba los costos de laparoscopia (16-18), como sexta posibilidad se calculó el incremento del costo bajo la contingencia de uso de trocar desechable en el $100 \%$ de los pacientes sometidos a laparoscopia.

Finalmente, dejando constantes los costos y modificando la variable efectividad, se planteó una última opción con los niveles de efectividad encontrados en la literatura científica $(11,18)$ reunidos en el metaanálisis de Olaya, et al. (19). No se analizaron situaciones en las que la efectividad fuera menor a la observada en alguna de las técnicas, ya que no se considera admisible evaluar si una técnica sigue siendo costoefectiva sacrificando el nivel de efectividad clínica encontrado.

\section{Resultados}

Se incluyeron 376 pacientes en el estudio, de los cuales, $220(58,5 \%)$ fueron intervenidos por técnica laparoscópica y $156(41,5 \%)$ por procedimiento abierto (cuadro 1). La edad promedio fue de 49,2 en el primer grupo y de 47,3 años en el segundo. En su mayoría, los pacientes intervenidos fueron mujeres.

El 99,4\% de las cirugías abiertas se hicieron en uno de los hospitales y en una mayor proporción fueron de carácter urgente, mientras que, de las laparoscópicas, el $60 \%$ se hizo en el otro hospital y en mayor proporción fueron programadas. Hubo menos cirugías en pacientes con ASA III y IV en ambos procedimientos. La prueba estadística de ji al cuadrado arrojó valores $p<0,05$ en todas 
Cuadro 1. Información demográfica

\begin{tabular}{|c|c|c|c|c|c|}
\hline \multirow{2}{*}{$\begin{array}{l}\text { Variable } \\
\text { Edad promedio (desviación estándar) }\end{array}$} & \multicolumn{2}{|c|}{$\begin{array}{l}\text { Laparoscopia } \\
\mathrm{n}=220(58,5 \%)\end{array}$} & \multicolumn{2}{|c|}{$\begin{array}{c}\text { Abierta } \\
\mathrm{n}=156(41,5 \%)\end{array}$} & \multirow{2}{*}{$\begin{array}{c}\mathbf{p} \\
0,268\end{array}$} \\
\hline & 49,2 & $(16,4)$ & 47,3 & $(15,6)$ & \\
\hline \multicolumn{6}{|l|}{ Sexo } \\
\hline Masculino & 62 & $(28,2)$ & 62 & $(39,7)$ & \multirow[t]{2}{*}{0,019} \\
\hline Femenino & 158 & $(71,8)$ & 94 & $(60,3)$ & \\
\hline \multicolumn{6}{|l|}{ Tipo de afiliación } \\
\hline Contributivo & 140 & $(73,6)$ & 154 & $(98,7)$ & \multirow[t]{4}{*}{$<0,001$} \\
\hline Prepagado & 70 & $(31,8)$ & 0 & $(0,0)$ & \\
\hline Particular & 9 & $(4,1)$ & 2 & $(1,3)$ & \\
\hline Vinculado & 1 & $(0,5)$ & 0 & $(0,0)$ & \\
\hline \multicolumn{6}{|l|}{ Tipo de cirugía } \\
\hline Programada & 130 & $(59,1)$ & 32 & $(20,5)$ & \multirow[t]{2}{*}{$<0,001$} \\
\hline Urgencias & 90 & $(40,9)$ & 124 & $(79,5)$ & \\
\hline \multicolumn{6}{|l|}{ ASA } \\
\hline I - II & 168 & $(76,4)$ & 89 & $(57,1)$ & \multirow[t]{2}{*}{$<0,001$} \\
\hline III - IV & 52 & $(23,6)$ & 67 & $(43,0)$ & \\
\hline \multicolumn{6}{|l|}{ Hospital } \\
\hline Hospital I & 88 & $(40,0)$ & 155 & $(99,4)$ & \multirow[t]{2}{*}{$<0,001$} \\
\hline Hospital II & 132 & $(60,0)$ & 1 & $(0,6)$ & \\
\hline
\end{tabular}

Cuadro 2. Odds ratio crudo y diferencia en medias (abierta Vs. laparoscopia)

\begin{tabular}{|c|c|c|c|c|}
\hline Variable & $\begin{array}{l}\text { Laparoscopia } \\
n=220(58,5 \%)\end{array}$ & $\begin{array}{c}\text { Abierta } \\
n=156 \\
(41,5 \%)\end{array}$ & $\mathbf{p}$ & $\begin{array}{l}\text { OR crudo* / } \\
\text { Diferencia de medias } \\
\text { (IC } 95 \% \text { ) }\end{array}$ \\
\hline Estancia hospitalaria en días (DE*) & $1,6 \quad(1,5)$ & $2,2 \quad(2,2)$ & 0,003 & $\begin{array}{c}0,60 \\
(0,2-1,0)\end{array}$ \\
\hline Duración del procedimiento en minutos (DE) & $89,7(41,1)$ & $67,6(24,6)$ & 0,001 & $\begin{array}{c}-22,1 \\
(-15,4)-(-28,8)\end{array}$ \\
\hline Complicaciones & $(6,4)$ & $21(13,5)$ & 0,020 & $\begin{array}{c}2,3 \\
(1,1-4,7) \\
2,0 \\
(0,9-4,4)^{\star}\end{array}$ \\
\hline Reincorporación de actividades cotidianas (DE) & $9,6(10,9)$ & $32,5(16,4)$ & $<0,001$ & $\begin{array}{c}22,9 \\
(19,8-26,0)\end{array}$ \\
\hline Ingreso a UCI & $(0,0)$ & $3(1,9)$ & 0,039 & No calculable \\
\hline
\end{tabular}

*OR ajustado; DE: desviación estándar; UCI: unidad de cuidado intensivo

las variables categóricas, indicando diferencias significativas en la distribución de las mismas entre ambos procedimientos.

En el cuadro 2 se observa que la estancia hospitalaria sin ajustar mostró diferencias significativas entre ambos procedimientos, siendo de 0,60 días más en la abierta $\left(\mathrm{IC}_{95 \%}: 0,2-1,0\right)$. La duración del procedimiento fue mayor en la cirugía laparoscópica $(p<0,001)$, mientras que el número de días de reincorporación de actividades, reportado por los pacientes, fue significativamente mayor en el procedimiento abierto $(p<0,001)$. La incapacidad clínica por norma siempre fue recomendada por 7 y 14 días para laparoscopia y abierta, respectivamente. El riesgo crudo de complicaciones fue mayor y significativo en la cirugía abierta $(p=0,02) y$, ajustado en un modelo de regresión logística, fue de $2,0\left(\mathrm{IC}_{95 \%}: 0,9-4,4\right)$, lo que indica que no existen diferencias significativas de riesgo de complicación entre el procedimiento abierto y el laparoscópico.

En total, se presentaron 25 complicaciones en 21 pacientes de colecistectomía abierta y 16 en 14 pacientes de laparoscopia. La complicación más frecuente fue el hemoperitoneo, que se presentó en 11 pacientes ( 6 de abierta y 5 de laparoscopia). La infección de la herida quirúrgica fue la segunda complicación más frecuente; se presentó en tres pacientes de procedimiento abierto y dos de laparoscopia. 
Cuadro 3. Duración del procedimiento (minutos) y valores ajustados

\begin{tabular}{lcc}
\hline Procedimiento & \multicolumn{2}{c}{ Tipo de cirugía } \\
& Programada & Urgencia \\
\hline Laparoscopia & 77,64 & 85,98 \\
Abierta & 58,50 & 65,73 \\
\hline
\end{tabular}

Estancia posoperatoria (días) y valores ajustados

\begin{tabular}{|c|c|c|}
\hline \multirow[t]{2}{*}{ Procedimiento } & \multicolumn{2}{|c|}{ Tipo de cirugía } \\
\hline & Programada & Urgencia \\
\hline Laparoscopia & 1,16 & 1,34 \\
\hline Abierta & 1,24 & 1,51 \\
\hline \multirow[t]{2}{*}{ Procedimiento } & \multicolumn{2}{|c|}{ ASA } \\
\hline & $I-\|$ & III-IV \\
\hline Laparoscopia & 1,17 & 1,44 \\
\hline Abierta & 1,32 & 1,64 \\
\hline
\end{tabular}

Al correr las variables "duración del procedimiento" y "estancia hospitalaria" en un modelo de regresión robusta, se observó que el tipo de cirugía y el ASA tuvieron asociación significativa con la variabilidad de aquellas, por lo que se presentan en el cuadro 3 los valores estimados, ajustados por estas últimas variables. Se observa que en cuanto a la duración del procedimiento, ésta fue mayor para la técnica laparoscópica, tanto en cirugía programada como de urgencia. Con respecto a la estancia hospitalaria, la cirugía abierta generó más tiempo posoperatorio en el hospital, tanto programada como de urgencia, así como en pacientes de menor y mayor gravedad.

En cuanto a los costos (en dólares estadounidenses), el promedio en colecistectomía laparoscópica desde la perspectiva de la IPS resultó ser menor con relación al procedimiento abierto (cuadro 4). De acuerdo con la razón de incremento del costo-efectividad, el ahorro que se obtendría por cada paciente sano adicional intervenido por laparoscopia, sería el equivalente a US\$7,4 (cuadro 4). La laparoscopia es menos costo-efectiva que la abierta, cuando se compara el mejor de los casos intervenidos por este último procedimiento con el caso más difícil sometido a laparoscopia, así como cuando se comparan los dos mejores casos en ambas técnicas y cuando la razón de incremento se calcula con la mediana como medida de tendencia central. En el resto de situaciones, la técnica laparoscópica genera un ahorro por unidad de efectividad adicional alcanzada.

En cuanto a la distribución de los costos, el servicio que más recursos demandó en los hospitales correspondió al del personal de sala de cirugía (cirujano, ayudante de cirugía y anestesiólogo): $34,79 \%$ de los costos para la cirugía abierta y 32,9 \% para la laparoscópica; la estancia posoperatoria ocupó el segundo lugar en costos: $19,6 \%$ en cirugía abierta y $15,1 \%$ en laparoscopia. La depreciación de equipos fue uno de los rubros que menor concentración de recursos presentó, y correspondió a $0,3 \%$ de los costos en la cirugía abierta y a 3,9\% en la laparoscópica. Los suministros generaron mayores recursos en la cirugía laparoscópica $(12,75 \%$ Vs. $5,63 \%)$ por el uso de elementos desechables, así como los derechos de sala $(14,0 \%$ Vs.10,4 $\%)$ debido a los mayores tiempos empleados en

Cuadro 4. Perspectiva IPS: costo promedio, efectividad, costo efectividad y análisis

\begin{tabular}{lccccccc}
\hline Escenarios & Costo & $\begin{array}{c}\text { Laparoscopia } \\
\text { Efectividad }\end{array}$ & $\mathbf{n}$ & Costo & $\begin{array}{c}\text { Abierta } \\
\text { Efectividad }\end{array}$ & $\mathbf{n}$ & RCEI* $^{*}$ \\
\hline Costo medio & 995 & 93,64 & 220 & 1.048 & 86,54 & 156 & -7.5 \\
Situación I & $4.098,6$ & 93,64 & 220 & 595,7 & 86,54 & 156 & 493,4 \\
Situación II & 653,1 & 93,64 & 220 & $7.842,5$ & 86,54 & 156 & $-1.012,6$ \\
Situación III & $4.098,6$ & 93,64 & 220 & $7.842,5$ & 86,54 & 156 & $-527,3$ \\
Situación IV & 653,1 & 93,64 & 220 & 595,7 & 86,54 & 156 & 8,1 \\
Situación V & 879 & 93,64 & 220 & 803,9 & 86,54 & 156 & 10,6 \\
Situación VI & $1.039,7$ & 93,64 & 220 & 1.048 & 86,54 & 156 & $-1,2$ \\
Situación VII & 995,4 & 95,60 & 220 & 1.048 & 93,20 & 156 & $-21,9$ \\
\hline
\end{tabular}

Situación I. Mejor situación para el procedimiento abierto comparado con el peor en el laparoscópico

Situación II. Mejor situación para el procedimiento laparoscópico comparado con el peor en el abierto

Situación III. Peor situación para ambos procedimientos

Situación IV. Mejor situación para ambos procedimientos

Situación V. Se calcula la razón de incremento de la costo-efectividad con el valor de la mediana

Situación VI. Uso de trocares, $100 \%$

Situación VII. Metaanálisis de Olaya, et al/19

${ }^{*} \mathrm{RCEI}$ : incremento de costo-fectividad (US\$/unidad de costo-efectividad) 
esta última. Tanto los medicamentos como los procedimientos, incluidos procedimientos médicos, imágenes diagnósticas, interconsultas de medicina especializada, visitas de valoración y estudio anatomopatológico, representaron una mayor proporción de costos en la cirugía abierta que en la laparoscópica (14,1 \% Vs. 11,6 \% y 12,6 \% Vs.9,8 $\%$, respectivamente).

Desde la perspectiva del paciente, se observó también un menor gasto promedio de bolsillo generado para los que fueron sometidos a laparoscopia y un ahorro obtenido por cada unidad adicional de efectividad. El gasto promedio de bolsillo incurrido por los pacientes del procedimiento abierto en los días posteriores a la cirugía y por causa de la misma, duplicó lo gastado por los del otro grupo (cuadro 5). El análisis de sensibilidad muestra que en todos los casos, exceptuando la primera posibilidad, el procedimiento laparoscópico generó menores costos para el paciente con relación al procedimiento de comparación (cuadro 5). La variable que más afecta la decisión en términos de costos, es el costo del procedimiento quirúrgico.

\section{Discusión}

Los resultados de este estudio se unen a los reportados en diversos tipos de evaluación económica que se han abordado en el ámbito mundial, dirigidos a determinar la validez de la colecistectomía laparoscópica. En ellos se han demostrado ventajas económicas a favor de ésta o similares a las del procedimiento abierto, mejor calidad de vida para ambos sexos en los distintos grupos de edades, menor cantidad de medicamentos utilizados para el control del dolor, menor estancia posoperatoria, recuperación más rápida, y retorno más temprano al trabajo y a la actividad normal (2-6).

Existen varios reportes en la literatura científica de estudios de costo-efectividad y de costo-utilidad, respecto a la situación planteada. A continuación se enuncian las características generales de algunos de ellos. En ningún estudio se plantea una evaluación con un horizonte temporal mayor de un año; (20-23) la perspectiva utilizada en la mayoría de los estudios evaluados, fueron del tercer pagador $(17,18,20,23-28)$ y algunos proponen la perspectiva social $(26,29-33)$. Recientemente, se han publicado estudios de costo-utilidad en los que se evalúan las posibilidades posoperatorias inmediatas y tardías; asimismo, en algunos se evalúa el efecto por QALY (Quality-Adjusted Life Years) de la intervención laparoscópica temprana y tardía, y se reportan datos importantes sobre las preferencias de los pacientes hacia una intervención temprana de la enfermedad (30-33). Con los QALY reportados se evalúa una disponibilidad de pago que oscila entre US\$10 a US\$16 millones por el manejo laparoscópico temprano de la enfermedad, evitando el compromiso de la calidad de vida y las complicaciones quirúrgicas mayores y de la enfermedad (28-33).

Para la interpretación de los resultados aquí presentados, es importante mencionar las ventajas y limitaciones propias de la metodología utilizada. Por una parte, al estimar cada componente de uso de recursos y derivar una unidad de costos para cada uno, el enfoque utilizado de microcostos es el más preciso (11). Además, parte de los estudios de evaluación económica hechos en el

Cuadro 5. Perspectiva paciente: costo promedio, efectividad, costo efectividad y análisis de sensibilidad (US\$)

\begin{tabular}{lrcccccc}
\hline Escenarios & Costo & $\begin{array}{c}\text { Laparoscopia } \\
\text { Efectividad }\end{array}$ & $\mathbf{n}$ & Costo & $\begin{array}{c}\text { Abierta } \\
\text { Efectividad }^{-}\end{array}$ & $\mathbf{n}$ & RCEI* $^{\star}$ \\
\hline Costo medio & 53,2 & 93,64 & 220 & 104,8 & 86,54 & 156 & $-7,3$ \\
Situación I & 582,8 & 93,64 & 220 & 0 & 86,54 & 156 & 82,1 \\
Situación II & 0 & 93,64 & 220 & 1593,3 & 86,54 & 156 & $-224,4$ \\
Situación III & 82,8 & 93,64 & 220 & 1593,3 & 86,54 & 156 & $-142,3$ \\
Situación IV & 0 & 93,64 & 220 & 0 & 86,54 & 156 & 0 \\
Situación IV & 17,2 & 93,64 & 220 & 76,2 & 86,54 & 156 & $-8,3$ \\
\hline
\end{tabular}

*Se calcula la razón de incremento del costo-efectividad con el valor de la mediana.

Situación I. Mejor situación para el procedimiento abierto comparado con el peor en el laparoscópico

Situación II. Mejor situación para el procedimiento laparoscópico comparado con el peor en el abierto

Situación III. Peor situación para ambos procedimientos

Situación IV. Mejor situación para ambos procedimientos

Situación V. Se calcula la razón de incremento del costo-efectividad con el valor de la mediana

${ }^{*}$ RCEl: incremento del costo-efectividad (US\$/unidad de costo efectividad) 
país, utilizan información reportada en la literatura científica, más de contextos externos que locales. Las conclusiones aquí planteadas provienen de una muestra de la población colombiana, con base en información primaria recolectada de manera prospectiva, desde el momento de la cirugía hasta un mes después de practicada. Aun cuando, al limitarse al contexto de dos hospitales del país, no permite generalizar los resultados a todos los prestadores que, probablemente, presenten variadas condiciones en la práctica regular (por ejemplo, el nivel de experiencia de los cirujanos), sí es evidente que la cirugía laparoscópica puede tener un impacto muy importante si se dan las condiciones que se han expuesto, como el entrenamiento de los cirujanos, así como aumentar y mejorar la práctica de la técnica quirúrgica que se ha evaluado.

Una de las argumentaciones que han reforzado omitir el uso de la laparoscopia en el contexto del sistema de salud colombiano, ha sido el costo de los equipos. Como se observó en este estudio, la depreciación de los equipos es uno de los rubros que menor concentración de recursos presenta. El uso de trocares desechables, bajo el supuesto de su utilización en todos los pacientes y con base en un protocolo de un trocar para cada tres pacientes, tampoco representó un incremento importante en el costo medio. Incluso en estas circunstancias, la técnica laparoscópica sigue siendo más costoefectiva que la abierta.

Otro aspecto que refuerza el uso de la técnica evaluada, es que los días de estancia hospitalaria posoperatoria, que representan una parte importante del costo total para el hospital, son menos en este procedimiento. Debe aclararse, en cuanto al costo de los equipos, que no se incluyeron equipos de esterilización de ninguno de los dos tipos de procedimiento y que, al utilizarse solamente el método de depreciación de equipos en línea recta, no se tuvo en cuenta el costo de oportunidad por el uso de los mismos. De igual manera, es importante mencionar que, a pesar de ser una variable que se debe considerar en el cálculo de los costos, el costo del tiempo gastado por los pacientes al recibir el tratamiento (por ejemplo, tiempos de espera y de movilización) no se calculó, ya que las alternativas analizadas, por ser intervenciones que no requieren tratamientos posteriores ni seguimiento durante amplios periodos, involucran gastos de tiempo similares y, por ende, su inclusión tendría un efecto insignificante sobre los resultados del análisis que, a nuestro juicio, no justifica el esfuerzo para su recolección y cálculo (12).

En cuanto a los costos de productividad de los pacientes, la exclusión en el cálculo del tiempo de incapacidad o convalecencia para el caso de este estudio, se justifica porque es un costo que en algunas ocasiones es asumido por el empleador $y$, en otras, por el paciente o por el asegurador. En vista de que este estudio se hizo únicamente desde la perspectiva del paciente y no desde la social, este último cálculo no se llevó a cabo. Sin embargo, debe tenerse en cuenta que, para análisis posteriores, la incapacidad, bien sea aquella legítimamente sentida por el paciente o la formal otorgada por el médico tratante, es un elemento muy importante para la decisión del tipo de procedimiento quirúrgico, considerando que en la cirugía abierta la incapacidad formulada por el médico representa el doble que en la laparoscópica.

Posiblemente, los estudios de estimación de costos desde el punto de vista social, en que se mide la producción perdida por día de recuperación, como se han hecho en otros países, incrementarían las ventajas de la colecistectomía laparoscópica sobre la abierta. También, es importante tener en cuenta que durante buena parte del tiempo en que se llevó a cabo el estudio, todavía la técnica evaluada no estaba incluida en el plan obligatorio de salud (POS) y, aun después de incluida, la colecistectomía laparoscópica seguía siendo una técnica menos generalizada y su enseñanza no se había expandido al grueso de los cirujanos del país. Esto lleva a sugerir que, a medida que este tratamiento se difunda y aumente la experiencia de los cirujanos, va a generar mejores resultados en cuanto a efectividad y, por lo tanto, en cuanto a costos frente a la técnica abierta, que durante más de 100 años ha sido el tratamiento estándar para la enfermedad litiásica vesicular (34). Además, en esta misma vía se espera que las tasas de ocupación en salas de cirugía por laparoscopia sean mayores y disminuya el costo por derechos de sala. Infortunadamente, esta tendencia no pudo observarse en el presente estudio por falta de disponibilidad de datos históricos en los hospitales incluidos. En el caso de las personas que ayudan en el cuidado del paciente (familiares o voluntarios), la aproximación preferida por algunos autores (12) es calcular el salario por hora de individuos que trabajan con características similares 0 , suponiendo que estos voluntarios no trabajan, haciendo una 
aproximación similar a la de amas de casa o desempleados. En el presente caso, se aproximó por salario mínimo y no por grupos de población, ya que no se contó con información sobre edad, sexo y otras características de los cuidadores.

El cálculo del tamaño de la muestra asumió la asignación de los sujetos de manera equitativa entre los dos grupos de estudio. Sin embargo, como el estudio no requirió el tamaño de muestra propuesto en su inicio, no se logró esta asignación 50/50; es necesario recalcar que este hecho podría comprometer el poder estadístico del estudio epidemiológico y debe tenerse en cuenta en el momento de evaluar los resultados.

Los principales hallazgos aquí dilucidados muestran que:

1) el riesgo de complicaciones para el paciente es mayor con la colecistectomía abierta; sin embargo, al ajustarlo, la diferencia deja de ser significativa;

2) en relación con las variables "estancia hospitalaria" y "duración de la cirugía", el procedimiento laparoscópico presenta ventajas en cuanto a la primera y desventajas en cuanto a la segunda;

3) la incapacidad reportada por los pacientes muestra resultados favorables importantes con la laparoscopia;

4) el costo directo de la colecistectomía laparoscópica es menor que el de la abierta, tanto para la IPS como para los pacientes;

5) no obstante lo anterior, según el análisis de sensibilidad y teniendo en cuenta que si grandes variaciones en la estimación de costos no generan cambios significativos en los resultados, éstos son juzgados como sólidos (17), al no mantenerse una razón negativa de incremento de la costo-efectividad o, por lo menos, cercana a cero en todos las posibilidades planteadas, se concluye que la técnica laparoscópica es tan costo-efectiva como la abierta.

La acción futura que debe seguir, en virtud de los hallazgos de este estudio, incluye la concientización del pagador sobre los resultados favorables de efectividad de la colecistectomía laparoscópica y similares en cuanto a costos, que permita un mayor favorecimiento de la población objeto de este tipo de cirugías. De la misma manera, deben intensificarse los talleres de capacitación del grueso número de cirujanos colombianos que aún no poseen la destreza o los equipos para su práctica.

\section{Agradecimientos}

Los autores agradecen a Adriana Córdoba, por la inclusión de pacientes, al igual que a Mauricio Gómez y Manuel Barrios, por el valioso trabajo en la recolección de información; a Andrés Gutiérrez, Germán Villegas, Jorge Millán y Lina Cadena, por los importantes aportes al procedimiento de costeo en los hospitales; a Diana Pinto, por los pertinentes comentarios a la metodología, y a Fabián Gil, por los importantes aportes para el análisis estadístico.

\section{Conflicto de intereses}

Ninguno declarado.

\section{Financiación}

Este estudio se realizó gracias a la financiación del Departamento Administrativo de Ciencia, Tecnología e Innovación, Colciencias.

\section{Referencias}

1. Berggren U, Zethraeus N, Arvidsson D, Haglund U, Jonsson B. A cost-minimization analysis of laparoscopic cholecystectomy versus open cholecystectomy. Am J Surg. 1996;172:305-10.

2. Bass EB, Pitt HA, Lillemoe KD. Cost-effectiveness of laparoscopic cholecystectomy versusopen cholecystectomy. Am J Surg. 1993;165:466-71.

3. Bosch F, Wehrman U, Saeger H, Kirch W. Laparoscopic or open conventional cholecystectomy: Clinical and economic considerations. Eur J Surg. 2002;168:270-7.

4. De Pouvourville G, Ribet-Reinhart N, Fendrick M, Houry $\mathbf{S}$, Testas P, Huguier M. A prospective comparison of costs and morbidity of laparoscopic versus open cholecystectomy. Hepatogastroenterology. 1997;44:35-9.

5. Zacks S, Sandler R, Rutledge R, Brown R. A populationbased cohort study comparing laparoscopic cholecystectomy and open cholecystectomy. Am J Gastroenterol. 2002;97:334-40.

6. Charlo T, Fernández $\mathbf{M}$, Tejido $\mathbf{C}$. Análisis de costes de la colecistectomía laparoscópica en comparación con la abierta. Rev Esp Enf Digest. 1995;87:449-52.

7. Moore JH, Rodríguez S, Roa A, Girón M, Sanabria $\mathbf{A}$, Rodríguez $\mathbf{P}$, et al. Colecistectomía laparoscópica ambulatoria: modelo de programa costo-eficiente de cirugía laparoscópica. Revista Colombiana de Cirugía. 2004;19:4353.

8. Olaya C, Carrasquilla G. Modelo de costo efectividad de la colecistectomía laparoscópica versus abierta. Bogotá: Fundación Santa Fe de Bogotá; 2004.

9. Hannan E, Imperato PJ, Nenner R, Harriet $\mathbf{S}$. Laparoscopic an open cholecystectomy in New York State: Mortality, complications, and choice of procedure. Surgery. 1999;125:223-31. 
10. Wolters $\mathbf{T}$, Wolf $\mathbf{H}$, Schroder T. ASA classification and perioperative variables as predictors of postoperative outcome. Br J Anaesth. 1996;77:217-22.

11. Drumond MF, O'Brien BJ, Stoddart GL, Torrance GW. Métodos para la evaluación económica de los programas de asistencia sanitaria. Segunda edición. Madrid: Ediciones Días de Santos S.A.; 2001.

12. Gold MR, Siegel JE, Russell LB, Weinstein MC. Costeffectiveness in health and medicine. New York: Oxford University Press; 1996. p. 176-220.

13. Petitti DB. Meta-analysis, decision analysis and costeffectiveness analysis. Methods for quantitative synthesis in medicine. Second edition. New York: Oxford University Press; 2000. p. 17.

14. Karnon J, Brown J. Selecting a decision model for economic evaluation: A case study and review. Health Care Manag Sci. 1998;1:133-40.

15. Gaitán H, Eslava-Schmalbach J, Gómez P. Cost effectiveness of diagnostic laparoscopy in reproductive aged females suffering from non-specific acute low abdominal pain. Rev Salud Pública. 2005;7:166-79.

16. Kesteloot K, Penninckx F. The cost and effects of open versus laparoscopic cholecystectomies. Health Econ. 1993;2:303-12.

17. Ruangsin S, Jaroonrach V, Petpichetchian C, Puttawibul P, Sunpaweravong S, Chewatanagongun S. Establishment the cost-effectiveness through set criteria of laparoscopic cholecystectomy. J Med Assoc Thai. 2010;93:789-93.

18. Srivastava A, Srinivas G, Misra MC, Pandav CS, Seenu V, Goyal A. Cost-effectiveness analysis of laparoscopic versus minilaparotomy cholecystectomy for gallstone disease. A randomized trial. Int J Technol Assess Health Care. 2001l;17:497-502.

19. Olaya C, Carrasquilla G. Meta-análisis de efectividad de la colecistectomía laparoscópica frente a la abierta. Revista Colombiana de Cirugía. 2006;21:104-15.

20. Rosen MJ, Malm JA, Tarnoff M, Zuccala K, Ponsky JL. Cost-effectiveness of ambulatory laparoscopic cholecystectomy. Surg Laparosc Endosc Percutan Tech. 2001;11:182-4.

21. Barkun JS, Caro JJ, Barkun AN, Trindade E. Costeffectiveness of laparoscopic and mini-cholecystectomy in a prospective randomized trial. Surg Endosc. 1995;9:1221-4.
22. McKellar DP, Johnson RM, Dutro JA, Mellinger J, Bernie WA, Peoples JB. Cost-effectiveness of laparoscopic cholecystectomy. Surg Endosc. 1995;9:158-62.

23. Mowschenson PM. Improving the cost-effectiveness of laparoscopic cholecystectomy. J Laparoendosc Surg. 1993;3:113-9.

24. Nicholl JP, Brazier JE, Milner PC, Westlake L, Kohler B, Williams BT, et al. Randomized controlled trial of costeffectiveness of lithotripsy and open cholecystectomy as treatments for gallbladder stones. Lancet. 1992;340:801-7.

25. Schirmer BD, Dix J. Cost effectiveness of laparoscopic cholecystectomy. J Laparoendosc Surg. 1992;2:145-50.

26. Pietrafitta JJ, Graner TJ, Nervick JM. Laparoscopic cholecystectomy: Cost effectiveness and quality enhancement. Int Surg. 1994;79:195-201.

27. Alinder G, Herlin P, Holmin T, Lindgren B. Routine operative cholangiography or pre-operative infusion cholangiography at elective cholecystectomy-a cost-effectiveness analysis. Acta Chir Scand. 1987;153:431-5.

28. Reder VA, Fineberg HV, Rosoff CB, White LS. Shorter length of stay for simple cholecystectomy: Cost-effectiveness of alternative strategies. Med Care. 1983;21:745-54.

29. Velu PP, Hor K, Leighton S, Yeoh SE, Duxbury M. Cost-utility and value-of-information analysis of early versus delayed laparoscopic cholecystectomy for acute cholecystitis. Br J Surg. 2010;97:1149-50.

30. Wilson E, Gurusamy K, Gluud C, Davidson BR. Cost-utility and value-of-information analysis of early versus delayed laparoscopic cholecystectomy for acute cholecystitis. $\mathrm{Br} \mathrm{J}$ Surg. 2010;97:210-9.

31. Clements WD. Commentary on cost-utility and value-ofinformation analysis of early versus delayed laparoscopic cholecystectomy for acute cholecystitis. $\mathrm{Br} \mathrm{J}$ Surg. 2010;97:219.

32. Mercer S. Letter 2: prospective randomized trial using cost-utility analysis of early versus delayed laparoscopic cholecystectomy for acute gallbladder disease. Br J Surg. 2009;96:1492-3.

33. Cervantes J. Historia de la colecistectomía por laparoscopia. En: Cervantes J, Patiño JF, editores. Cirugía laparoscópica y toracoscópica. México: McGraw-Hill; 1997. p. 45-50. 